\title{
Universal Polynomials for Severi Degrees of Toric Surfaces
}

\author{
Federico Ardila $\|$ and Florian Block非 \\ ${ }^{1}$ Department of Mathematics, San Francisco State University, USA \\ ${ }^{2}$ Mathematics Institute, University of Warwick, United Kingdom
}

\begin{abstract}
The Severi variety parameterizes plane curves of degree $d$ with $\delta$ nodes. Its degree is called the Severi degree. For large enough $d$, the Severi degrees coincide with the Gromov-Witten invariants of $\mathbb{C P}^{2}$. Fomin and Mikhalkin (2009) proved the 1995 conjecture that for fixed $\delta$, Severi degrees are eventually polynomial in $d$.

In this paper, we study the Severi varieties corresponding to a large family of toric surfaces. We prove the analogous result that the Severi degrees are eventually polynomial as a function of the multidegree. More surprisingly, we show that the Severi degrees are also eventually polynomial "as a function of the surface".

Our strategy is to use tropical geometry to express Severi degrees in terms of Brugallé and Mikhalkin's floor diagrams, and study those combinatorial objects in detail. An important ingredient in the proof is the polynomiality of the discrete volume of a variable facet-unimodular polytope.

Résumé. La variété de Severi paramétrise les courbes planes de degré $d$ avec $\delta$ noeuds. Son degré s'appelle le degré de Severi. Pour $d$ assez grand, les degrés de Severi cö̈ncident avec les invariants de Gromov-Witten de $\mathbb{C P}^{2}$. Fomin et Mikhalkin (2009) ont prouvé une conjecture de 1995 que pour $\delta$ fixé, les degrés de Severi sont à terme des polynômes en $d$.

Nous étudions les variétés de Severi correspondant à une large famille de surfaces toriques. Nous prouvons le résultat analogue que les degrés de Severi sont à terme des fonctions polynomiales du multidegré. De manière plus surprenante, nous montrons que les degrés de Severi sont à terme des polynômes en tant que "fonction de la surface".

Notre stratégie est d'utiliser la géométrie tropicale pour exprimer les degrés de Severi en fonction des "floor diagrams" de Brugallé et Mikhalkin, et d'utiliser ces objets combinatoires en détail. Un autre ingrédient important de la pruve est la polynomialité du volume discret d'un polytope face-unimodulaire variable.
\end{abstract}

Keywords: Enumerative geometry, toric surfaces, Gromov-Witten theory, Severi degrees, node polynomials

\section{Introduction and Main Theorems}

\subsection{Severi degrees and node polynomials for $\mathbb{C P}^{2}$.}

A $\delta$-nodal curve is a reduced (not necessarily irreducible) curve having $\delta$ simple nodes and no other singularities. The Severi degree $N^{d, \delta}$ is the degree of the Severi variety parameterizing degree $d \delta$-nodal curves in the complex projective plane $\mathbb{C P}^{2}$. In other words, $N^{d, \delta}$ is the number of such curves through an appropriate number of points in general position. For $d \geq \delta+2, N^{d, \delta}$ equals the Gromov-Witten invariant $N_{d,\left(\begin{array}{c}d-1 \\ 2\end{array}\right)-\delta}$.

Severi varieties were introduced around 1915 by Enriques [Enr12] and Severi [Sev21] , and have received considerable attention. Much later, in 1986, Harris [Har86] achieved a celebrated breakthrough by proving their irreducibility.

In 2009, Fomin and Mikhalkin [FM10, Theorem 5.1] proved Di Francesco and Itzykson's 1995 conjecture [DFI95] that, for a fixed number of nodes $\delta$, the Severi degree $N^{d, \delta}$ becomes a polynomial $N_{\delta}(d)$ in the degree, for $d \geq 2 \delta$. We will call $N_{\delta}(d)$ the node polynomial following Kleiman-Piene [KP04].

\footnotetext{
${ }^{\dagger}$ Partially supported by the National Science Foundation CAREER Award DMS-0956178 and the National Science Foundation Grant DMS-0801075.

${ }^{\ddagger}$ Partially supported by the National Science Foundation Grant DMS-055588 and a Rackham Fellowship. 


\subsection{Severi degrees and node polynomials for toric surfaces.}

The purpose of this paper is to generalize the previous results to the context of counting curves on a large family of (possibly non-smooth) toric surfaces $\mathrm{S}(\mathbf{c})$, which includes $\mathbb{C P}^{1} \times \mathbb{C P}^{1}$ and Hirzebruch surfaces. A new and interesting feature of our results is that the Severi degree $N_{\mathrm{S}(\mathbf{c})}^{\mathbf{d}, \delta}$ of such a toric surface $\mathrm{S}(\mathbf{c})$ is a polynomial not only as a function of the degree $\mathbf{d}$, but also as a function of $\mathbf{c}$, i.e., as a "function of the surface" itself. We now state our results more precisely.

Notation 1.1 A polygon $P$ is said to be h-transverse if it has integer coordinates and every edge has slope 0 , $\infty$, or $\frac{1}{k}$ for some integer $k$. Let $d^{t}$ and $d^{b}$ be the lengths of the top and bottom edges of $P$, if they exist (and 0 if they don't exist). Let the edges on the right side of the polygon, listed clockwise from top to bottom, have directions $\left(c_{1}^{r},-1\right), \ldots,\left(c_{n}^{r},-1\right)$ and lattice lengths $d_{1}^{r}, \ldots, d_{n}^{r}$, so $c_{1}^{r}>\cdots>c_{n}^{r}$. Let the edges on the left side of the polygon, listed counterclockwise from top to bottom, have directions $\left(c_{1}^{l},-1\right), \ldots,\left(c_{m}^{l},-1\right)$ and lattice lengths $d_{1}^{l}, \ldots, d_{m}^{l}$, so $c_{1}^{l}<\cdots<c_{m}^{l}$. Notice that $d^{t}+\sum_{i} c_{i}^{r} d_{i}^{r}-d^{b}-\sum_{j} c_{j}^{l} d_{j}^{l}=0$.

Denote $\mathbf{c}^{r}=\left(c_{1}^{r}, \ldots, c_{n}^{r}\right), \mathbf{d}^{r}=\left(d_{1}^{r}, \ldots, d_{n}^{r}\right), \mathbf{c}^{l}=\left(c_{1}^{l}, \ldots, c_{m}^{l}\right), \mathbf{d}^{l}=\left(d_{1}^{l}, \ldots, d_{m}^{l}\right)$, and $\mathbf{c}=$ $\left(\mathbf{c}^{r} ; \mathbf{c}^{l}\right), \mathbf{d}=\left(d^{t} ; \mathbf{d}^{r} ; \mathbf{d}^{l}\right)$. Finally, denote $\Delta(\mathbf{c}, \mathbf{d}):=P$. Observe that $\mathbf{c}$ is the set of slopes of the nonvertical rays in the normal fan of $\Delta(\mathbf{c}, \mathbf{d})$.

Figure 1 shows the polygon $\Delta(\mathbf{c}, \mathbf{d})$, together with its normal fan, for $\mathbf{c}=((3,1,0,-1) ;(-2,0,1,2))$ and $\mathbf{d}=(1 ;(1,2,1,1) ;(1,1,1,2))$.
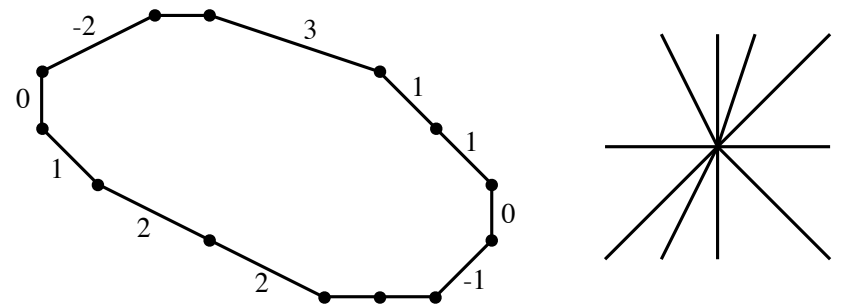

Fig. 1: An $h$-transverse polygon and its normal fan.

The normal fan of the polygon $\Delta(\mathbf{c}, \mathbf{d})$ consists of the outward rays centered at the origin and perpendicular to the sides. This fan determines a projective toric surface $\mathrm{S}(\mathbf{c}$ ) (which only depends on $\mathbf{c}$ and whether $d^{t}$ and $d^{b}$ are zero). Additionally, the polygon itself determines an ample line bundle $\mathcal{L}_{\mathbf{c}}(\mathbf{d})$ on $\mathrm{S}(\mathbf{c})$; let $\left|\mathcal{L}_{\mathbf{c}}(\mathbf{d})\right|$ be the complete linear system of divisors on $\mathrm{S}(\mathbf{c})$ corresponding to $\mathcal{L}_{\mathbf{c}}(\mathbf{d})$.

When we count curves on $\mathrm{S}(\mathbf{c})$, we will loosely think of $\mathbf{c}$ as the surface where our curves live, and $\mathbf{d}$ as their multidegree. This is motivated by the case when $\Delta(\mathbf{c}, \mathbf{d})=\operatorname{conv}\{(0,0),(m, 0),(0, m)\}$. In this case the toric surface is $\mathbb{C P}^{2}$, and the linear system $\left|\mathcal{L}_{\mathbf{c}}(\mathbf{d})\right|$ parameterizes the degree $m$ curves on $\mathbb{C P}^{2}$.

Given a positive integer $\delta$, the Severi variety is the closure of the set of $\delta$-nodal curves in $\left|\mathcal{L}_{\mathbf{c}}(\mathbf{d})\right|$. Its degree is the Severi degree $N_{\mathrm{S}(\mathbf{c})}^{\mathbf{d}, \delta}$. This number also counts:

- the $\delta$-nodal curves in $\left|\mathcal{L}_{\mathbf{c}}(\mathbf{d})\right|$ which pass through given $\left|\Delta \cap \mathbb{Z}^{2}\right|-1-\delta$ generic points in $\mathrm{S}(\mathbf{c})$, and

- the $\delta$-nodal curves in the torus $\left(\mathbb{C}^{*}\right)^{2}$ defined by polynomials with Newton polygon $\Delta(\mathbf{c}, \mathbf{d})$ which go through given $\left|\Delta \cap \mathbb{Z}^{2}\right|-1-\delta$ generic points in $\left(\mathbb{C}^{*}\right)^{2}$.

Our main result is that, for a fixed number of nodes $\delta$, the Severi degree $N_{\mathrm{S}(\mathbf{c})}^{\mathbf{d}, \delta}$ is a polynomial in both $\mathbf{c}$ and $\mathbf{d}$, provided $\mathbf{c}$ and $\mathbf{d}$ are sufficiently large and "spread out", in the precise sense defined below.

Theorem 1.2 (Polynomiality of Severi degrees.)

Fix $m, n \geq 1$ and $\delta \geq 1$. There is a universal and combinatorially defined polynomial $p_{\delta}(\mathbf{c}, \mathbf{d})$ such that the Severi degree $N_{\mathrm{S}(\mathbf{c})}^{\mathrm{d}, \delta}$ is given by

$$
N_{\mathrm{S}(\mathbf{c})}^{\mathbf{d}, \delta}=p_{\delta}(\mathbf{c}, \mathbf{d})
$$

for any sufficiently large and spread out $\mathbf{c} \in \mathbb{Z}^{m+n}$ and $\mathbf{d} \in \mathbb{Z}_{\geq 0}^{m+n+1}$. 
More precisely, the result holds if we assume, in Notation 1.1 that:

$$
\begin{aligned}
d^{t}, d^{b} & \geq \delta \\
d^{t}+c_{1}^{r}-c_{1}^{l}, d^{b}+c_{n}^{r}-c_{m}^{l} & \geq 2 \delta \\
d_{i}^{r}, d_{j}^{l} & \geq \delta+1 \quad(1 \leq i \leq n, 1 \leq j \leq m), \\
\left|\left(d_{1}^{r}+\cdots+d_{i}^{r}\right)-\left(d_{1}^{l}+\cdots+d_{j}^{l}\right)\right| & \geq \delta+2 \quad(1 \leq i \leq n-1,1 \leq j \leq m-1), \\
c_{i}^{r}-c_{i+1}^{r}, c_{j+1}^{l}-c_{j}^{l} & \geq \delta+1 \quad(1 \leq i \leq n-1,1 \leq j \leq m-1) .
\end{aligned}
$$

As special cases, we obtain polynomiality results for curve counts on $\mathbb{C P}^{1} \times \mathbb{C P}^{1}$, and Hirzebruch surfaces. Similar results hold for toric surfaces arising from polygons with one or no horizontal edges, such as $\mathbb{C P}^{2}$; see Remark [AB10, Remark 5.2].

The restriction in Theorem 1.2 to toric surfaces of h-transverse polygons is a technical assumption necessary for our proof. Floor diagrams, our main combinatorial tools, are (as of now) only defined in this situation. We expect, however, that similar results hold for arbitrary toric surfaces.

\subsection{The relationship with Göttsche's Conjecture.}

Our work is closely related to Göttsche's Conjecture [Göt98, Conjecture. 2.1], but the precise relationship still requires clarification. Göttsche conjectured the existence of universal polynomials $T_{\delta}(x, y, z, t)$ that compute the Severi degree for any smooth projective algebraic surface $S$ and any sufficiently ample line bundle $\mathcal{L}$ on $S$. According to the conjecture, the number of $\delta$-nodal curves in the linear system $|\mathcal{L}|$ through an appropriate number of points is given by evaluating $T_{\delta}$ at the four topological numbers $\mathcal{L}^{2}, \mathcal{L} K_{S}, K_{S}^{2}$ and $C_{2}(S)$. Here $K_{S}$ denotes the canonical bundle, $C_{1}$ and $C_{2}$ represent Chern classes, and $L M$ denotes the degree of $C_{1}(L) \cdot C_{1}(M)$ for line bundles $L$ and $M$. Recently, Tzeng proved Göttsche's Conjecture [Tze10].

If the toric surface $\mathrm{S}(\mathbf{c})$ is smooth, then all four topological numbers mentioned above are polynomials in $\mathbf{c}$ and $\mathbf{d}$. In that case, Tzeng's proof of Göttsche's conjecture implies that, for fixed $\delta$, the Severi degrees $N_{\mathrm{S}(\mathbf{c})}^{\mathbf{d}, \delta}$ are given by a universal polynomial in $\mathbf{c}$ and $\mathbf{d}$, provided that $\mathcal{L}_{\mathbf{c}}(\mathbf{d})$ is $(5 \delta-1)$-ample.

Göttsche's conjecture does not imply our results because the toric surfaces considered in Theorem 1.2 are almost never smooth. The surface $\mathrm{S}(\mathbf{c})$ is smooth precisely when any two adjacent rays in the normal fan span the lattice $\mathbb{Z}^{2}$. This happens if and only if $c_{1}^{r}-c_{2}^{r}=\cdots=c_{n-1}^{r}-c_{n}^{r}=1$ and $c_{1}^{l}-c_{2}^{l}=\cdots=$ $c_{m-1}^{l}-c_{m}^{l}=-1$.

It is natural to ask for a generalization of the four topological numbers to singular surfaces so that Göttsche's universal polynomial $T_{\delta}(x, y, z, t)$ specializes to the polynomial of Theorem 1.2 In essence, we do not know how to do this and can only speculate. One could pass to MacPherson's Chern class to define $C_{2}(S)$ for any singular surface $S$. For toric surfaces, $C_{2}(S)$ could also be defined via the combinatorial formula for the Chern polynomial of a toric variety. Generally, $C_{1}\left(K_{S}\right)$ is defined for any projective variety [Ful84, Section 3]. However, we checked that $T_{\delta}(x, y, z, t)$, when evaluated at any of the proposed sets of numbers, gives a different polynomial. Alternatively, evaluating $T_{\delta}(x, y, z, t)$ at the topological numbers of a smooth resolution of $\mathrm{S}(\mathbf{c})$ does not yield the desired result either.

Still, the Severi degrees are uniformly given by a polynomial in $\mathbf{c}$ and $\mathbf{d}$, provided $\mathbf{d}$ is sufficiently large. This possibly suggests a generalization of Göttsche's conjecture to a class of singular algebraic surfaces.

\subsection{Outline}

This paper is organized as follows. In Section 2 we discuss how to compute Severi degrees of " $h$-transverse" toric surfaces in terms of floor diagrams, generalizing Brugallé and Mikhalkin's method. In Section 3 we generalize Fomin and Mikhalkin's "template decomposition" of a floor diagram, and express Severi degrees in terms of templates. The resulting formula is intricate and not obviously polynomial. In Section 4, we express Severi degrees as a finite sum, where each summand is a "discrete integral" of a polynomial function over a variable polytopal domain. This allows us to prove their eventual polynomiality. For more details and complete proofs see [AB10]. 


\section{Counting curves with floor diagrams}

In this section we review the floor diagrams of Brugallé and Mikhalkin [BM07, BM09] associated to curves on toric surfaces which come from $h$-transverse polygons. We will introduce them using notation inspired by Fomin and Mikhalkin [FM10] who discussed floor diagrams in the planar case.

\subsection{Counting curves via floor diagrams.}

We now explain how the Severi degrees $N_{\mathrm{S}(\mathbf{c})}^{\mathrm{d}, \delta}$ can be computed combinatorially, following Brugalle and Mikhalkin's work. [BM07, BM09] For the rest of the paper we assume that $\Delta=\Delta(\mathbf{c}, \mathbf{d})$ is $h$-transverse. Define the multiset $D_{r}$ of right directions of $\Delta$ to be the multiset containing each right direction $c_{i}^{r}$ repeated $d_{i}^{r}$ times. Define $D_{l}$ analogously. The cardinality of $D_{r}$ (or, equivalently, of $D_{l}$ ) is the height of $\Delta$.

Example 2.1 For the polygon $\Delta$ of Figure 1 , the multisets of left directions and right directions are $D_{l}=$ $\{-2,0,1,2,2\}$ and $D_{r}=\{3,1,1,0,-1\}$ and the upper edge length is $d^{t}=1$.

Now we define the combinatorial objects which, weighted correctly, compute $N^{\Delta, \delta}$.

Definition $2.2 A \Delta$-floor diagram $\mathcal{D}$ consists of:

- two permutations ${ }^{(\mathrm{i})}\left(l_{1}, \ldots l_{M}\right)$ and $\left(r_{1}, \ldots r_{M}\right)$ of the multisets $D_{l}$ and $D_{r}$ of left and right directions of $\Delta$, and a sequence $\left(s_{1}, \ldots s_{M}\right)$ of non-negative integers such that $s_{1}+\cdots+s_{M}=d^{t}$,

- a graph on vertices $\{1, \ldots, M\}$, possibly with multiple edges, with edges directed $i \rightarrow j$ if $i<j$, and

- edge weights $w(e) \in \mathbb{Z}_{>0}$ for all edges e such that for every vertex $j$,

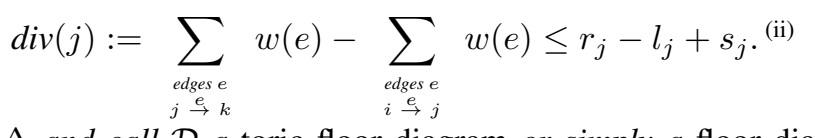

Sometimes we will omit $\Delta$ and call $\mathcal{D}$ a toric floor diagram or simply a floor diagram. When a floor diagram has $\mathbf{l}=\left(l_{1}, \ldots, l_{M}\right), \mathbf{r}=\left(r_{1}, \ldots, r_{M}\right), \mathbf{s}=\left(s_{1}, \ldots, s_{M}\right)$, we will call it an $(\mathbf{l}, \mathbf{r}, \mathbf{s})$-floor diagram. We will also call $\mathbf{a}:=\left(d^{t}, \mathbf{r}-\mathbf{l}\right)$ the divergence sequence, because in Definition 2.4 we will add some edges to obtain a diagram $\tilde{\mathcal{D}}$ with this vertex divergence sequence, and it is this new diagram that we will mostly be working with.

Example 2.3 Figure 2 shows a toric floor diagram corresponding, with $D_{l}=\{0,0,0,0\}, D_{r}=\{1,1,1,0\}$, and $I_{0}=1$. We place the vertices on a line in increasing order and omit the (left-to-right) edge directions.

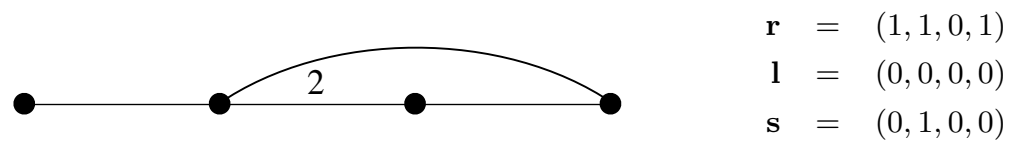

Fig. 2: A toric floor diagram.

A floor diagram $\mathcal{D}$ is connected if its underlying graph is. Notice that in [BM09] floor diagrams are necessarily connected; we don't require that. The genus of $\mathcal{D}$ is the genus $g(\mathcal{D})$ of the underlying graph (or the first Betti number of the underlying topological space). If $\mathcal{D}$ is connected its cogenus is given by

$$
\delta(\mathcal{D})=\left|\operatorname{int}(\Delta) \cap \mathbb{Z}^{2}\right|-g(\mathcal{D}),
$$

where $\operatorname{int}(\Delta)$ denotes the interior of the polygon $\Delta$. This definition is motivated by the fact that an irreducible algebraic curve of genus $g$ with $\delta$ nodes and Newton polygon $\Delta$ satisfies $\delta+g=\left|\operatorname{int}(\Delta) \cap \mathbb{Z}^{2}\right|$. Via the correspondence between algebraic curves and floor diagrams (see [BM09]) these notions literally correspond to the respective analogues for algebraic curves. Connectedness corresponds to irreducibility. Lastly, a floor diagram $D$ has multiplicity $\mu(\mathcal{D})=\prod_{\text {edges } e} w(e)^{2}$.

To enumerate algebraic curves via floor diagrams we need to count certain markings of these diagrams.

(i) The permutations of a multiset are counted without repetition. For instance, the multiset $\{1,1,2\}$ has three permutations: $(1,1,2),(1,2,1),(2,1,1)$.

(ii) This inequality will become clearer when we define the markings of a floor diagram. 
Definition 2.4 A marking of a floor diagram $\mathcal{D}$ is defined by the following four step process.

Step 1: For each vertex $j$ of $\mathcal{D}$, create $s_{j}$ new indistinguishable vertices and connect them to $j$ with new edges directed towards $j$.

Step 2: For each vertex $j$ of $\mathcal{D}$, create $r_{j}-l_{j}+s_{j}-\operatorname{div}(j)$ new indistinguishable vertices and connect them to $j$ with new edges directed away from $j$. This makes the divergence of vertex $j$ equal to $r_{j}-l_{j}$ for $1 \leq j \leq M$.

Step 3: Subdivide each edge of the original floor diagram $\mathcal{D}$ into two directed edges by introducing a new vertex for each edge. The new edges inherit their weights and orientations. Denote the resulting graph $\tilde{\mathcal{D}}$.

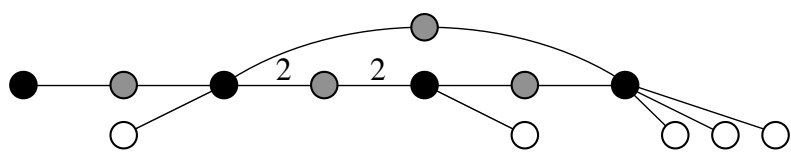

$$
\begin{aligned}
\mathbf{r} & =(1,1,0,1) \\
\mathbf{l} & =(0,0,0,0) \\
\mathbf{s} & =(0,1,0,0) \\
\mathbf{r}-\mathbf{l} & =(1,1,0,1)
\end{aligned}
$$

Fig. 3: The result of applying Steps 1-3 to Figure2

Step 4: Linearly order the vertices of $\tilde{\mathcal{D}}$ extending the order of the vertices of the original floor diagram $\mathcal{D}$ such that, as before, each edge is directed from a smaller vertex to a larger vertex.

The extended graph $\tilde{\mathcal{D}}$ together with the linear order on its vertices is called a marked floor diagram, or $a$ marking of the original floor diagram $\mathcal{D}$.

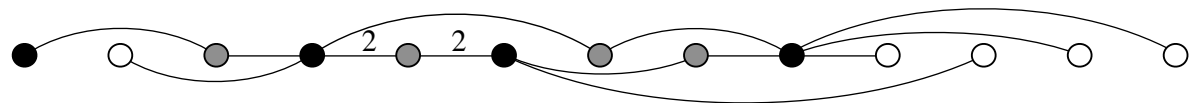

$\mathbf{r}=(1,1,0,1), \mathbf{l}=(0,0,0,0), \mathbf{s}=(0,1,0,0), \quad \mathbf{r}-\mathbf{l}=(1,1,0,1)$

Fig. 4: A marking of the floor diagram of Figure 2

Keeping in mind that we introduced indistinguishable vertices in Steps 1 and 2, we need to count marked floor diagrams up to equivalence. Two such $\tilde{\mathcal{D}}_{1}, \tilde{\mathcal{D}}_{2}$ are equivalent if $\tilde{\mathcal{D}}_{1}$ can be obtained from $\tilde{\mathcal{D}}_{2}$ by permuting edges without changing their weights; i.e., if there exists an automorphism of weighted graphs which preserves the vertices of $\mathcal{D}$ and maps $\tilde{\mathcal{D}}_{1}$ to $\tilde{\mathcal{D}}_{2}$. The number of markings $\nu(\mathcal{D})$ is the number of marked floor diagrams $\tilde{\mathcal{D}}$ up to equivalence.

Example 2.5 Let us compute $\nu(\mathcal{D})$ for the floor diagram of Figure 2 by counting the possible linear orderings of Figure 3 Modulo isomorphism, the ordering of ten of the vertices is fixed. The leftmost lower white vertex can be inserted in three places. The top gray vertex can be placed in 4 positions. For two of them, the second white vertex can be placed in 6 positions, while for the other two it can be placed in 7 positions. Therefore $\nu(\mathcal{D})=3(2 \cdot 6+2 \cdot 7)=78$.

Let $\mathcal{D}$ be a floor diagram. Let $V(\mathcal{D})=\cup_{i=1}^{t} V_{i}$ be the partition of the vertices of $\mathcal{D}$ given by the connected components of $\mathcal{D}$, and let $\mathcal{D}_{1}, \ldots, \mathcal{D}_{t}$ be the corresponding (connected) floor diagrams. Define $h$-transverse polygons $\Delta_{i}$ (for $1 \leq i \leq t$ ) by the collections $\left\{\left(l_{j}, r_{j}, s_{j}\right)\right\}_{j \in V_{i}}$, where in each such collection the index $j$ runs over the vertices $j$ in $V_{i}$. Define $\delta(\mathcal{D})=\sum_{i} \delta\left(\mathcal{D}_{i}\right)+\mathcal{M}\left(\Delta_{1}, \ldots, \Delta_{t}\right)$. (This definition is motivated by Bernstein's theorem [Ber75], see [AB10, Section 2.3] for details.) It is not hard to write an explicit expression for $\delta(\mathcal{D})$.

Theorem 2.6 For any h-transverse polygon $\Delta$ and any $\delta \geq 0$ the Severi degree $N^{\Delta, \delta}$ is given by

$$
N^{\Delta, \delta}=\sum \mu(\mathcal{D}) \nu(\mathcal{D}),
$$

summing over all $\Delta$-floor diagrams $\mathcal{D}$ of cogenus $\delta$.

Equation Severi1] is the first in a series of combinatorial formulas for the Severi degree $N^{\Delta, \delta}$, which we use to prove the eventual polynomiality of $N^{\Delta, \delta}$. While the right hand side is certainly combinatorial, it is unmanageable in several ways. The first difficulty is that the indexing set is terribly complicated. The following section provides a first step towards gaining control over it. 


\section{Template decomposition of floor diagrams and Severi degrees}

We now introduce a decomposition of the floor diagrams of Section 2 into "basic building blocks", called templates. This extends earlier work of Fomin and Mikhalkin [FM10] who did this in the planar case.

\subsection{Templates.}

Definition 3.1 [FM10 Definition 5.6]. A template $\Gamma$ is a directed graph on vertices $\{0, \ldots, l\}$, where $l \geq 1$, with possibly multiple edges and edge weights $w(e) \in \mathbb{Z}_{>0}$, satisfying:

1. If $i \stackrel{e}{\rightarrow} j$ is an edge then $i<j$.

2. Every edge $i \stackrel{e}{\rightarrow} i+1$ has weight $w(e) \geq 2$. (No "short edges".)

3. For each vertex $j, 1 \leq j \leq l-1$, there is an edge "covering" it, i.e., there exists an edge $i \stackrel{e}{\rightarrow} k$ with $i<j<k$.

Every template $\Gamma$ comes with some numerical data associated to it, which will play an important role later. Its length $l(\Gamma)$ is the number of vertices minus 1 . The product of squares of the edge weights is its multiplicity $\mu(\Gamma)$. Its cogenus $\delta(\Gamma)$ is

$$
\delta(\Gamma)=\sum_{\substack{e \\ i \rightarrow j}}[(j-i) w(e)-1] .
$$

For $1 \leq j \leq l(\Gamma)$ let $\varkappa_{j}=\varkappa_{j}(\Gamma)$ denote the sum of the weights of edges $i \stackrel{e}{\rightarrow} k$ with $i<j \leq k$, which we can think of as the flow over the midpoint between $j-1$ and $j$. If $a_{j}(\Gamma)$ denotes the divergence of $\Gamma$ at vertex $j$, then $a_{j}(\Gamma)=\varkappa_{j+1}-\varkappa_{j}$, so we can also think of $\varkappa_{j}$ as the cumulative divergence to the left of $j$. Lastly, set

$$
\varepsilon_{0}(\Gamma)= \begin{cases}1 & \text { if all edges starting at } 0 \text { have weight } 1, \\ 0 & \text { otherwise. }\end{cases}
$$

Likewise, define $\varepsilon_{1}(\Gamma)$ by instead considering all edges arriving at $l$. See [AB10, Figure 6] for a list of all templates $\Gamma$ with $\delta(\Gamma) \leq 2$. Note that, for any $\delta$, there are only finitely many templates with cogenus $\delta$.

\subsection{Decomposing a floor diagram into templates.}

We now show how to decompose a floor diagram $\mathcal{D}$ on vertices $1, \ldots, M$ into templates. Recall that for each vertex $j$ of $\mathcal{D}$ we record a tuple of integers $\left(l_{j}, r_{j}, s_{j}\right)$.

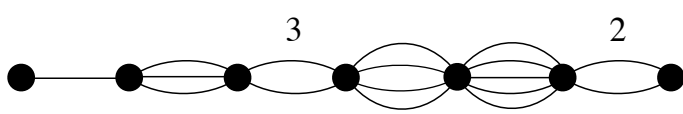

$\mathbf{r}=(1,1,1,0,1,0,0), \mathbf{l}=(0,0,0,0,0,0,0), \mathbf{s}=(0,1,0,0,0,0,0)$

Fig. 5: A floor diagram.

First, we add a vertex $0(<1)$ to $\mathcal{D}$, along with $s_{j}$ new edges of weight 1 from 0 to $j$ for each $1 \leq$ $j \leq M$. Then we add a vertex $M+1(>M)$, together with $r_{j}-l_{j}+s_{j}-\operatorname{div}(j)$ new edges of weight 1 from $i$ to $M+1$ for each $1 \leq j \leq M$. The vertex divergence sequence of the resulting diagram $\mathcal{D}^{\prime}$ is $\left(d^{t}, r_{1}-l_{1}, \ldots, r_{M}-l_{M},-d^{b}\right)$. We drop the (superfluous) last entry from this sequence and as before we say $\left(d^{t}, \mathbf{r}-\mathbf{l}\right)$ is the divergence sequence.

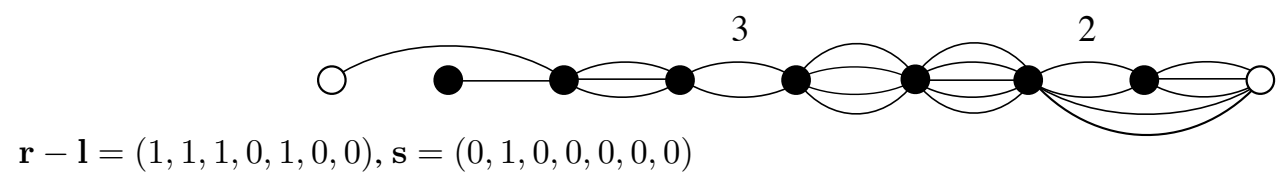

Fig. 6: The floor diagram of Figure 5 with additional initial and final vertices. 
Now remove all short edges from $\mathcal{D}^{\prime}$, that is, all edges of weight 1 between consecutive vertices. The result is an ordered collection of templates $\boldsymbol{\Gamma}=\left(\Gamma_{1}, \ldots, \Gamma_{m}\right)$, listed left to right. We also keep track of the initial vertices $k_{1}, \ldots, k_{m}$ of these templates.

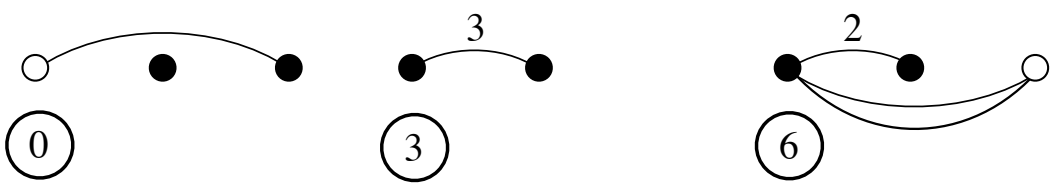

Fig. 7: The template decomposition of the floor diagram of Figure 5

Conversely, given the collection of templates $\boldsymbol{\Gamma}=\left(\Gamma_{1}, \ldots, \Gamma_{m}\right)$, the starting points $k_{1}, \ldots, k_{m}$, and the divergence sequence $\mathbf{a}=\left(d^{t}, \mathbf{r}-\mathbf{l}\right)$, this process is easily reversed. To recover $\mathcal{D}^{\prime}$, we first place the templates in their correct starting points in the interval $[1, \ldots, M]$, and draw in all the short edges that we removed from $\mathcal{D}^{\prime}$ from left to right. More precisely, to change the divergences from $a_{j}(\boldsymbol{\Gamma})$ (iii) to $a_{j}$, we need to add $\left(a_{0}-a_{0}(\boldsymbol{\Gamma})\right)+\cdots+\left(a_{j-1}-a_{j-1}(\boldsymbol{\Gamma})\right)=\left(a_{0}+\cdots+a_{j-1}-\varkappa_{j}(\boldsymbol{\Gamma})\right)$ short edges between $j-1$ and $j$. Finally, we remove the first and last vertices and their incident edges to obtain $\mathcal{D}$.

Given a divergence sequence $\mathbf{a}$ the possible starting points of the templates in a collection $\boldsymbol{\Gamma}=\left(\Gamma_{1}, \ldots, \Gamma_{m}\right)$ are restricted by $\mathbf{a}$. More precisely, the valid sequences of starting points $\mathbf{k}=\left(k_{1}, \ldots, k_{m}\right)$ of $\Gamma_{1}, \ldots, \Gamma_{m}$ are the ones in the set $A(\boldsymbol{\Gamma}, \mathbf{a})$, consisting of vectors $\mathbf{k} \in \mathbb{N}^{m}$ such that

- $k_{1} \geq 1-\varepsilon_{0}\left(\Gamma_{1}\right)$,

- $k_{i+1} \geq k_{i}+l\left(\Gamma_{i}\right)$ for all $i=1, \ldots, m-1$,

- $k_{m} \leq M-l\left(\Gamma_{m}\right)+\varepsilon_{1}\left(\Gamma_{m}\right)$, and

- $a_{0}+\cdots+a_{k_{i}+j-1}-\varkappa_{j}\left(\Gamma_{i}\right) \geq 0$ for $i=1, \ldots, m$, and $j=1, \ldots, l\left(\Gamma_{i}\right)$.

The first three inequalities guarantee that the templates fit in the interval $[1, \ldots, M]$ without overlapping. The last condition guarantees that the numbers of edges we need to add are non-negative. Notice that, for fixed $\mathbf{a}$, if $\delta(\mathcal{D})=0$ (i.e., if $\mathcal{D}$ is the unique floor diagram with only short edges and $s_{i}=0$ for $i \geq 2$ ) then $A(\boldsymbol{\Gamma}, \mathbf{a})$ is empty as the decomposition removes all edges. Due to this abnormality we exclude the case $\delta=0$ in the sequel, though it is not hard to see that $N^{\Delta, 0}=1$ for all $\Delta$.

We summarize the previous discussion in a proposition.

Proposition 3.2 Let $M \geq 1$, and let $\mathbf{l}, \mathbf{r} \in \mathbb{Z}^{M}, \mathbf{s} \in \mathbb{N}^{M}$. Let $d^{t}=s_{1}+\cdots+s_{M}$ and $\mathbf{a}=\left(d^{t}, \mathbf{r}-\mathbf{l}\right)$. The procedure of template decomposition is a bijection between the $(\mathbf{l}, \mathbf{r}, \mathbf{s})$-floor diagrams and the pairs $(\boldsymbol{\Gamma}, \mathbf{k})$ of a collection of templates $\boldsymbol{\Gamma}$ and a valid sequence of starting points $\mathbf{k} \in A(\boldsymbol{\Gamma}, \mathbf{a})$.

\subsection{Multiplicity, cogenus, and markings.}

Now we show that the multiplicity, cogenus, and markings of a floor diagram behave well under template decomposition.

If a floor diagram $\mathcal{D}$ has template decomposition $\boldsymbol{\Gamma}$, then clearly $\mu(\mathcal{D})=\prod_{i=1}^{m} \mu\left(\Gamma_{i}\right)$. Define the reversal sets $\operatorname{Rev}(\mathbf{r})$ of the sequences $\mathbf{r}$ and $\mathbf{l}$ by

$$
\operatorname{Rev}(\mathbf{r})=\left\{1 \leq i<j \leq M: r_{i}<r_{j}\right\}, \quad \operatorname{Rev}(\mathbf{l})=\left\{1 \leq i<j \leq M: l_{i}>l_{j}\right\} .
$$

The asymmetry is due to the fact that the "natural" order for $\mathbf{r}$ is the weakly decreasing one, while for $\mathbf{l}$ it is the weakly increasing one. Define the cogenus of the pair $(\mathbf{l}, \mathbf{r})$ as

$$
\delta(\mathbf{l}, \mathbf{r})=\sum_{(i, j) \in \operatorname{Rev}(\mathbf{r})}\left(r_{j}-r_{i}\right)+\sum_{(i, j) \in \operatorname{Rev}(-\mathbf{l})}\left(l_{i}-l_{j}\right) .
$$

\footnotetext{
(iii) We are denoting by $a_{j}(\boldsymbol{\Gamma})$ the divergence of vertex $j$ in the template $\Gamma_{i}$ containing it. Similarly, $\varkappa_{j}(\boldsymbol{\Gamma})=\varkappa_{j}\left(\Gamma_{i}\right)=a_{0}(\boldsymbol{\Gamma})+$ $\cdots+a_{j}(\boldsymbol{\Gamma})=a_{k_{i}}\left(\Gamma_{i}\right)+\cdots+a_{j}\left(\Gamma_{i}\right)$.
} 
Given a collection of templates $\boldsymbol{\Gamma}=\left(\Gamma_{1}, \ldots, \Gamma_{m}\right)$ we abbreviate the sum over their cogenera by $\delta(\boldsymbol{\Gamma}):=$ $\sum_{i=1}^{m} \delta\left(\Gamma_{i}\right)$. The template decomposition is cogenus preserving, in the sense that (for details see [AB10])

$$
\delta(\mathcal{D})=\delta(\boldsymbol{\Gamma})+\delta(\mathbf{l}, \mathbf{r})
$$

Let $\mathcal{D}$ be a floor diagram with divergence sequence $\mathbf{a}=\left(a_{0}, \ldots, a_{M}\right)=\left(d^{t}, \mathbf{r}-\mathbf{l}\right)$. For each template $\Gamma$ and each non-negative integer $k$ (for which $(3.1)$ is non-negative for all $j$ ) let $\Gamma_{(\mathbf{a}, k)}$ denote the graph obtained from $\Gamma$ by first adding

$$
a_{0}+a_{1}+\cdots+a_{k+j-1}-\varkappa_{j}(\Gamma)
$$

short edges connecting $j-1$ to $j$, for $1 \leq j \leq l(\Gamma)$ (so that the vertices now have divergences $a_{k}, \ldots, a_{k+l(\Gamma)}$ ), and then subdividing each edge of the resulting graph by introducing one new vertex for each edge. Let $P_{\Gamma}(\mathbf{a}, k)$ be the number of linear extensions (up to equivalence) of the vertex poset of the graph $\Gamma_{(\mathbf{a}, k)}$ extending the vertex order of $\Gamma$. Then

$$
\nu(\mathcal{D})=\prod_{i=1}^{m} P_{\Gamma_{i}}\left(\mathbf{a}, k_{i}\right)=: P_{\Gamma}(\mathbf{a}, \mathbf{k})
$$

With this machinery the Severi degree $N^{\Delta, \delta}$ can be computed solely in terms of templates. We conclude from Theorem 2.6. Proposition 3.2, and the previous observations in this section:

Proposition 3.3 For any h-transverse polygon $\Delta$ and $\delta \geq 1$ the Severi degree $N^{\Delta, \delta}$ is given by

$$
N^{\Delta, \delta}=\sum_{(\mathbf{1}, \mathbf{r}): \delta(\mathbf{l}, \mathbf{r}) \leq \delta} \sum_{\Gamma: \delta(\boldsymbol{\Gamma})=\delta-\delta(\mathbf{1}, \mathbf{r})}\left(\prod_{i=1}^{m} \mu\left(\Gamma_{i}\right) \sum_{\mathbf{k} \in A(\boldsymbol{\Gamma}, \mathbf{a})} P_{\boldsymbol{\Gamma}}(\mathbf{a}, \mathbf{k})\right)
$$

where the first sum is over all permutations $\mathbf{l}=\left(l_{1}, \ldots, l_{M}\right)$ and $\mathbf{r}=\left(r_{1}, \ldots, r_{M}\right)$ of the left and right directions $D_{l}$ and $D_{r}$ of $\Delta$ with $\delta(\mathbf{l}, \mathbf{r}) \leq \delta$, and the second sum is over template collections $\boldsymbol{\Gamma}$ of cogenus $\delta-\delta(\mathbf{l}, \mathbf{r})$. As before, we denote the upper edge length $d^{t}$ of $\Delta$ by $a_{0}$, and write $a_{i}=r_{i}-l_{i}$ for $1 \leq i \leq M$.

Severi2 improves Severi1) by removing the unwieldy divergence condition on floor diagrams. However, eventual polynomiality is still far from clear.

\section{Polynomiality of Severi degrees}

We will now use (Severi2) to sketch a proof of our main theorem: the polynomiality of the Severi degrees for toric surfaces given by sufficiently large $h$-transverse polygons. We do this in two steps. First we discuss the case of first-quadrant polygons. The proof of this special case exhibits essentially all the features of the general case.The arguments easily adapt to the general case (see [AB10, Section 5]).

Notation 4.1 We say that an h-transverse polygon $\Delta=\Delta(\mathbf{c}, \mathbf{d})$ is a first-quadrant polytope if $\mathbf{c}^{l}=\mathbf{0}=$ $(0, \ldots, 0)$ and $\mathbf{c}^{r} \geq \boldsymbol{0}$. We will then omit $\mathbf{c}^{l}$ and $\mathbf{d}^{l}$ from the notation and write $\Delta(\mathbf{c}, \mathbf{d})=\Delta\left(\mathbf{c}^{r},\left(d^{t} ; \mathbf{d}^{r}\right)\right)=$ $\Delta\left(\left(c_{1}, \ldots, c_{n}\right),\left(d_{0} ; d_{1}, \ldots, d_{n}\right)\right)$. The corresponding floor diagrams have $M=d_{1}+\cdots+d_{n}$ vertices. The multisets of left and right directions, and upper edge length are

$$
D_{l}=\{0, \ldots, 0\}, \quad D_{r}=\{\underbrace{c_{1}, \ldots, c_{1}}_{d_{1}}, \ldots, \underbrace{c_{n}, \ldots, c_{n}}_{d_{n}}\}, \quad d_{0} .
$$

Then $\mathbf{l}=\mathbf{0}$ and $\mathbf{a}=\left(d_{0}, \mathbf{r}\right)$. We write $\delta(\mathbf{r})=\delta(\mathbf{l}, \mathbf{r})$.

For example, Figure 8 shows the polygon $\Delta((1,0),(1 ; 3,1))$ which has right directions 1 and 0 with respective lengths 3 and 1 , and upper edge length equal to 1 . Here $D_{r}=\{1,1,1,0\}$.

Remark 4.2 In this section we will assume that $\Delta(\mathbf{c}, \mathbf{d})$ is a first-quadrant h-transverse polygon. We will also assume that

$$
d_{0} \geq \delta, \quad d_{0}+c_{1} \geq 2 \delta, \quad d_{1}, \ldots, d_{n} \geq \delta+1
$$

and will simply say that $\mathbf{d}$ is large enough to describe these inequalities. Throughout most of the section we we will hold $\mathbf{c}$ constant and let $\mathbf{d}$ vary. (When we let $\mathbf{c}$ vary, we will say so explicitly.) 

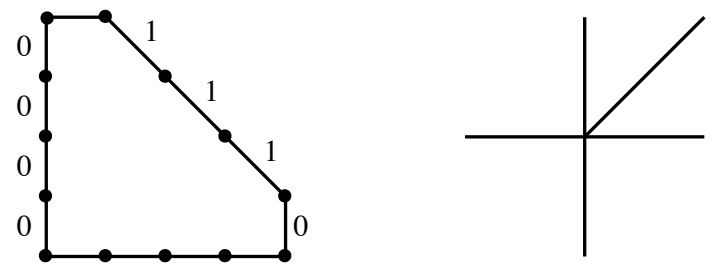

Fig. 8: The first-quadrant polygon $\Delta((1,0),(1 ; 3,1))$ and its normal fan.

Severi2 now reads:

$$
N^{\Delta, \delta}=\sum_{\mathbf{r}: \delta(\mathbf{r}) \leq \delta} \sum_{\Gamma: \delta(\boldsymbol{\Gamma})=\delta-\delta(\mathbf{r})}\left(\prod_{i=1}^{m} \mu\left(\Gamma_{i}\right) \sum_{\mathbf{k} \in A(\boldsymbol{\Gamma}, \mathbf{a})} P_{\boldsymbol{\Gamma}}(\mathbf{a}, \mathbf{k})\right)
$$

(Severi2’)

To show that (Severi2') yields an eventual polynomial in $\mathbf{c}$ and $\mathbf{d}$, our first problem is that the index set of the first sum is hard to control: as $\mathbf{c}$ and $\mathbf{d}$ vary, the index set of permutations $\mathbf{r}$ such that $\delta(\mathbf{r}) \leq \delta$ varies quite delicately with them. In particular, these permutations can be arbitrarily long. In turn, the index set of the second sum depends very sensitively on the value of $\delta(\mathbf{r})$. These problems are solved by presenting a more compact encoding of $\mathbf{r}$.

\subsection{From permutations to swaps.}

Let us organize the permutations $\mathbf{r}$ of $D_{r}$ of cogenus less than or equal $\delta$ in a way which is uniform for large $\mathbf{c}$ and $\mathbf{d}$. Observe that, if $\mathbf{d}$ is large enough, then such a permutation cannot contain a reversal of $c_{i}$ and $c_{j}$ for $i \geq j+2$. This is because the minimum "divergence cost" of reversing $c_{i-1}$ and $c_{i+1}$ is $d_{i} \min \left\{c_{i}-c_{i+1}, c_{i-1}-c_{i}\right\} \geq d_{i}>\delta$.

This observation allows us to encode such a permutation $\mathbf{r}$ into $n-1$ sequences of 1 s and -1 s which, for each $1 \leq i \leq n-1$, record the relative positions between the $c_{i} \mathrm{~s}$ and the $c_{i+1} \mathrm{~s}$.

Example 4.3 Suppose $\mathbf{c}=(5,3,2,1), \mathbf{d}=(0,4,6,4,3)$, and $\mathbf{r}=55335353233212121$. This permutation decomposes into three sequences of $1 s$ and -1 s as follows:

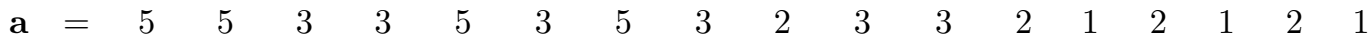

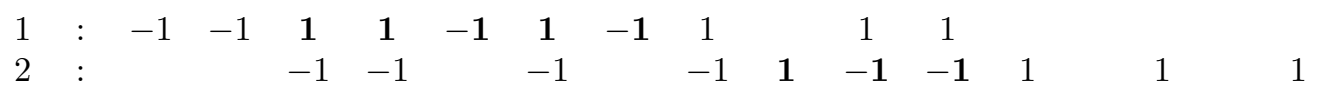

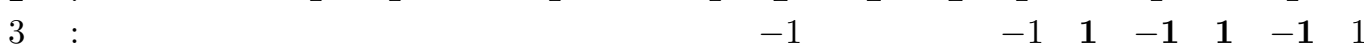

$$
\begin{aligned}
& \pi_{1}=(1,1,-1,1,-1), \quad \pi_{2}=(1,-1,-1), \quad \pi_{3}=(1,-1,1,-1)
\end{aligned}
$$

To achieve uniformity among different sequence lengths, we delete all initial -1 s and all final 1 s in each such sequence. The result is a swap, which we define to be a sequence of -1 s and 1 s which (is empty or) starts with a 1 and ends with a -1 .

We have encoded a permutation $\mathbf{r}$ into a sequence of $n-1$ swaps $\pi=\left(\pi_{1}, \ldots, \pi_{n-1}\right)$. Conversely, if we know $\mathbf{c}$ and $\mathbf{d}$ we can easily recover $\mathbf{r}=: \pi(\mathbf{c}, \mathbf{d})$ from $\pi$. Using the encoding of permutations into swaps, we now replace the first sum in (Severi2') by a sum over swaps. Let the number of inversions $\operatorname{inv}(\pi)$ of a swap $\pi$ be

$$
\operatorname{inv}(\pi)=\#\left\{(i, j) \in \mathbb{Z}^{2}: 1 \leq i<j \leq n-1 \text { and } \pi(i)>\pi(j)\right\} .
$$

It is easy to see that $\delta(\mathbf{r})=\sum_{i=1}^{n-1} \operatorname{inv}\left(\pi_{i}\right)\left(c_{i}-c_{i+1}\right)$. We obtain that, for large $\mathbf{d}$,

$$
N^{\Delta, \delta}=\sum_{\pi} \sum_{\boldsymbol{\Gamma}: \delta(\boldsymbol{\Gamma})=\delta-\delta(\mathbf{r})}\left(\prod_{i=1}^{m} \mu\left(\Gamma_{i}\right) \sum_{\mathbf{k} \in A(\boldsymbol{\Gamma}, \mathbf{a}) \cap \mathbb{Z}^{m}} P_{\boldsymbol{\Gamma}}(\mathbf{a}, \mathbf{k})\right)
$$

where the first sum is now over all sequences $\pi=\left(\pi_{1}, \ldots, \pi_{n-1}\right)$ of swaps with $\sum_{i=1}^{n-1} \operatorname{inv}\left(\pi_{i}\right)\left(c_{i}-c_{i+1}\right) \leq$ $\delta, \mathbf{r}=\pi(\mathbf{c}, \mathbf{d}), \mathbf{a}=\left(d_{0}, \mathbf{r}\right)$ and the other sums are as before. 
For fixed c, the first sum in (Severi3') is finite and its index set is independent of $\mathbf{d}$. Also, for each $\pi$ in that index set, $\delta(\mathbf{r})$ is independent of $\mathbf{d}$, and hence so is the set of templates $\boldsymbol{\Gamma}$ in the second sum. The difficulty encountered in Severi2') is resolved.

If $\mathbf{c}$ is variable this observation still applies, under the additional assumption that $\mathbf{c}$ grows quickly enough that $c_{i}-c_{i+1}>\delta$ for all $i$. In that case, the first sum will only include the trivial swap sequence $\pi$ where every swap is empty, and then the index set of the second sum will still be independent of $\mathbf{d}$, and also of $\mathbf{c}$.

In Severi3' we have expressed $N^{\Delta, \delta}$ as a weighted sum of finitely many contributions of the form

$$
N_{\pi, \boldsymbol{\Gamma}}^{\Delta, \delta}:=\sum_{\mathbf{k} \in A(\boldsymbol{\Gamma}, \mathbf{a}) \cap \mathbb{Z}^{m}} P_{\boldsymbol{\Gamma}}(\mathbf{a}, \mathbf{k}),
$$

where $\mathbf{a}=\left(d_{0}, \pi(\mathbf{c}, \mathbf{d})\right)$. Our final goal is to show that, for fixed $\delta, \boldsymbol{\Gamma}$, and $\pi$, and for large $\mathbf{d}$, this function varies piecewise polynomially in $\mathbf{c}$ and $\mathbf{d}$. We will do it over the course of the Sections 4.2-4.4 by showing that $A(\boldsymbol{\Gamma}, \mathbf{a})$ is a variable polytope and $P_{\boldsymbol{\Gamma}}(\mathbf{a}, \mathbf{k})$ is piecewise polynomial, and then recurring to some facts about such discrete integrals.

\subsection{Polytopality of $A(\Gamma, \mathbf{a})$ and piecewise polynomiality of $P_{\Gamma}(\mathbf{a}, \mathbf{k})$.}

Our next key propositions state that, for large enough $\mathbf{c}$ and $\mathbf{d}$, the innermost index set $A(\Gamma, \mathbf{a}) \cap \mathbb{Z}^{m}$ of Severi3] is the set of lattice points in a polytope, over which we then discretely integrate a piecewise polynomial (for the proofs see [AB10]).

Proposition 4.4 Let $\pi=\left(\pi_{1}, \ldots, \pi_{n-1}\right)$ be a fixed sequence of swaps and let $\boldsymbol{\Gamma}=\left(\Gamma_{1}, \ldots, \Gamma_{m}\right)$ be a fixed collection of templates. Let $\left(c_{1}>\cdots>c_{n}\right) \in \mathbb{Z}_{\geq 0}^{n}$ and $\mathbf{d}=\left(d_{0} ; d_{1}, \ldots, d_{n}\right) \in \mathbb{Z}_{>0}^{n+1}$ be variable and assume that $d_{0} \geq \delta(\boldsymbol{\Gamma}), d_{0}+c_{1} \geq 2 \delta(\boldsymbol{\Gamma})$. Let $\mathbf{a}=\left(d_{0}, \mathbf{r}\right)=\left(d_{0}, \pi(\mathbf{c}, \mathbf{d})\right)$. Then $A(\boldsymbol{\Gamma}, \mathbf{a})$ is the set of lattice points in a polytope whose facet directions are fixed, and whose facet parameters are linear functions of $d_{0}, \ldots, d_{n}$.

Proposition 4.5 Let $\pi=\left(\pi_{1}, \ldots, \pi_{n-1}\right)$ be a fixed collection of swaps and let $\boldsymbol{\Gamma}=\left(\Gamma_{1}, \ldots, \Gamma_{m}\right)$ be a fixed collection of templates. Let $\mathbf{c}=\left(c_{1}>\cdots>c_{n}\right) \in \mathbb{Z}_{>0}^{n}$ and $\mathbf{d}=\left(d_{0} ; d_{1}, \ldots, d_{n}\right) \in \mathbb{Z}_{\geq 0}^{n+1}$ be variable and $\mathbf{a}=\left(d_{0}, \mathbf{r}\right)=\left(d_{0}, \pi(\mathbf{c}, \mathbf{d})\right)$. Let $\mathbf{k} \in A(\boldsymbol{\Gamma}, \mathbf{a}) \cap \mathbb{Z}^{m}$ be variable. Then the function $P_{\boldsymbol{\Gamma}}(\overline{\mathbf{a}}, \mathbf{k})$ is piecewise polynomial in $\mathbf{c}, \mathbf{d}$ and $\mathbf{k}$. The domains of polynomiality are faces of a hyperplane arrangement.

\subsection{Discrete integrals of polynomials over polytopes.}

We saw that $A(\boldsymbol{\Gamma}, \mathbf{a}) \cap \mathbb{Z}^{m}$ is the set of lattice points in a polytope with fixed facet directions, and whose facet parameters are linear functions of $\mathbf{d}$. Since this set only depends on $\mathbf{d}$, we relabel it $A(\boldsymbol{\Gamma}, \mathbf{d}) \cap \mathbb{Z}^{m}$. Furthermore, we saw that $P_{\boldsymbol{\Gamma}}(\mathbf{a}, \mathbf{k})$ is a piecewise polynomial function of $\mathbf{c}, \mathbf{d}$, and $\mathbf{k}$, whose domains of polynomiality are cut out by a hyperplane arrangement $\mathcal{A}$. The equations of this arrangement have fixed normal directions, and parameters which are linear functions of $\mathbf{d}$ and $\mathbf{k}$. It follows that

$$
N_{\pi, \boldsymbol{\Gamma}}^{\Delta, \delta}=\sum_{F} \sum_{\mathbf{k} \in(A(\boldsymbol{\Gamma}, \mathbf{d}) \cap F)^{\circ} \cap \mathbb{Z}^{m}} P_{\boldsymbol{\Gamma}}^{F}(\mathbf{c}, \mathbf{d}, \mathbf{k}),
$$

summing over the faces $F$ of $\mathcal{A}$, where each $P_{\Gamma}^{F}$ is a polynomial. Here $Q^{o}$ denotes the relative interior of $Q$, i.e., the interior of $Q$ with respect to its affine span. We get:

$$
N^{\Delta, \delta}=\sum_{\pi, \boldsymbol{\Gamma}, F} \sum_{\mathbf{k} \in(A(\boldsymbol{\Gamma}, \mathbf{d}) \cap F)^{\circ} \cap \mathbb{Z}^{m}} P_{\boldsymbol{\Gamma}}^{F}(\mathbf{c}, \mathbf{d}, \mathbf{k}) .
$$

This is a somewhat messy expression, but the point is that there is a finite number of choices for $\pi, \boldsymbol{\Gamma}$, and $F$, and these choices are independent of $\mathbf{d}$. Now we just need to prove the polynomiality of the inner sum, which is a discrete integral of a polynomial function over a variable open polytope.

To do so, we invoke some results on discrete integrals. Given a polytope $Q \subset \mathbb{R}^{m}$ and a function $f: \mathbb{R}^{m} \rightarrow \mathbb{R}$, we define the discrete integral of $f$ over $Q$ to be

$$
\sum_{q \in Q \cap \mathbb{Z}^{m}} f(q)
$$


Recall that an $m$-polytope is simple if every vertex is contained in exactly $m$ edges. It is integral if all its vertices have integer coordinates. A facet translation of a polytope $P=\Pi_{X}(\mathbf{y})=\left\{\mathbf{k} \in \mathbb{R}^{m}: X \mathbf{k} \leq \mathbf{y}\right\}$ is a polytope of the form $\Pi_{X}\left(\mathbf{y}^{\prime}\right)=\left\{\mathbf{k} \in \mathbb{R}^{m}: X \mathbf{k} \leq \mathbf{y}^{\prime}\right\}$ for $\mathbf{y}^{\prime} \in \mathbb{R}^{l}$, obtained by translating the facets of $P$. We assume that $X$ is an integer matrix and say $\Pi_{X}\left(\mathbf{y}^{\prime}\right)$ is an integer facet translation if $\mathbf{y}^{\prime} \in \mathbb{Z}^{l}$. Say that the matrix $X$ is unimodular, and that $P$ is facet-unimodular, if every maximal minor has determinant $-1,0$, or 1 . In this case, every integer facet translation $\Pi_{X}\left(\mathbf{y}^{\prime}\right)$ has integral vertices by Cramer's rule.

The values of $\mathbf{y}^{\prime}$ for which $\Pi_{X}\left(\mathbf{y}^{\prime}\right)$ and $P$ are combinatorially equivalent form an open cone in $\mathbb{R}^{m}$; its closure is the deformation cone of $P$. The corresponding polytopes are deformations of $P$. [Pos09, PRW08]

Recall that a quasipolynomial function on a lattice $\Lambda$ is a function which is polynomial on each coset of some finite sublattice $\Lambda^{\prime} \subseteq \Lambda$. Results like the following are known, although we have not found in the literature the precise statement that we need:

Lemma 4.6 Consider a variable polytope with fixed facet directions, and facet parameters which vary linearly as a function of a vector $\mathbf{d}$; i.e.,

$$
\Pi_{X}(Y \mathbf{d})=\left\{\mathbf{k} \in \mathbb{R}^{m}: X \mathbf{k} \leq Y \mathbf{d}\right\}
$$

where $X \in \mathbb{Z}^{l \times m}$ and $Y \in \mathbb{Z}^{l \times n}$ are fixed $l \times m$ and $l \times n$ matrices, and $\mathbf{d} \in \mathbb{R}^{n}$ is a variable vector. Let $f(\mathbf{c}, \mathbf{d}, \mathbf{k})$ be a polynomial function of $\mathbf{c} \in \mathbb{R}^{n}, \mathbf{d} \in \mathbb{R}^{n}$, and $\mathbf{k} \in \mathbb{R}^{m}$, and let

$$
g(\mathbf{c}, \mathbf{d})=\sum_{\mathbf{k} \in \Pi_{X}(\mathbf{y}) \cap \mathbb{Z}^{m}} f(\mathbf{c}, \mathbf{d}, \mathbf{k}), \quad g^{o}(\mathbf{c}, \mathbf{d})=\sum_{\mathbf{k} \in \Pi_{X}(\mathbf{y})^{\circ} \cap \mathbb{Z}^{m}} f(\mathbf{c}, \mathbf{d}, \mathbf{k}) .
$$

Then $g(\mathbf{c}, \mathbf{d})$ and $g^{o}(\mathbf{c}, \mathbf{d})$ are piecewise polynomial functions of $\mathbf{c}$ and $\mathbf{d}$. The domains of quasipolynomiality are given by linear conditions in $\mathbf{d}$. More concretely, these functions are quasipolynomial when restricted to those $\mathbf{d}$ for which the polytope $\Pi_{X}(Y \mathbf{d})$ has a fixed combinatorial type.

Furthermore, if $X$ is unimodular, then $g(\mathbf{c}, \mathbf{d})$ and $g^{o}(\mathbf{c}, \mathbf{d})$ are piecewise polynomial.

Proof: See [AB10].

\subsection{Polynomiality of Severi degrees.}

The eventual polynomiality of Severi degrees in the special case of first-quadrant polygons now follows from the previous discussions.

Theorem 4.7 (Polynomiality of first-quadrant Severi degrees.)

Fix $n \geq 1$ and $\delta \geq 1$. There is a universal polynomial $p_{\delta}(\mathbf{c}, \mathbf{d})$ such that the Severi degree $N_{\mathrm{S}(\mathbf{c})}^{\mathrm{d}, \delta}$ is given by

$$
N_{\mathrm{S}(\mathbf{c})}^{\mathbf{d}, \delta}=p_{\delta}(\mathbf{c}, \mathbf{d}) .
$$

for any $\mathbf{c}=\left(c_{1}>\cdots>c_{n}\right) \in \mathbb{Z}^{n}$ and $\mathbf{d} \in \mathbb{Z}_{\geq 0}^{n+1}$ such that $c_{i}-c_{i+1} \geq \delta+1, d_{i} \geq \delta+1$ for all $i, d_{0} \geq \delta$ and $d_{0}+c_{1} \geq 2 \delta$.

Proof: (Sketch, for a complete proof see[AB10].)

Step 1. Piecewise quasipolynomiality. In Severi4 , for each choice for $\pi, \boldsymbol{\Gamma}$, and $F, P_{\boldsymbol{\Gamma}}^{F}(\mathbf{c}, \mathbf{d}, \mathbf{k})$ is polynomial in $\mathbf{c}, \mathbf{d}$, and $\mathbf{k}$ and $A(\boldsymbol{\Gamma}, \mathbf{d}) \cap F$ is polytopal with fixed facet directions and facet parameters which are linear in $\mathbf{d}$ (thanks to Section 4.2. Lemma 4.6 then shows that $N^{\Delta, \delta}$ is piecewise quasipolynomial in $\mathbf{c}$ (which is constant here) and $\mathbf{d}$.

Step 2. Quasipolynomiality. On can show, with some effort, that all large enough $\mathbf{d}$ lie in the same domain of quasipolynomiality. Thus, $N^{\Delta, \delta}$ is a quasipolynomial.

Step 3. Polynomiality. Since the polytopes $A(\boldsymbol{\Gamma}, \mathbf{d}) \cap F$ are facet-unimodular, $N^{\Delta, \delta}$ is polynomial.

Remark 4.8 Equation (Severi3] gives an algorithm to compute the polynomial $p_{\delta}(\mathbf{c}, \mathbf{d})$; see [AB10] for more details and explicit computations.

The proof for general $h$-transverse polygons (Theorem 1.2 follows along similar lines as the outlined proof of Theorem 4.7, see [AB10]. 


\section{Acknowledgements}

Acknowledgements. We thank the referee for helpful suggestions and Benoît Bertrand and Erwan Brugallé for clarifying some details about floor diagrams. The second author thanks Eugene Eisenstein, José González, and Alan Stapledon for explaining some facts about toric surfaces, and Bill Fulton for valuable comments on the subject. Part of this work was accomplished at the MSRI (Mathematical Sciences Research Institute) in Berkeley, CA, USA, during the Fall 2009 semester in tropical geometry. We would like to thank MSRI for their hospitality.

\section{References}

[AB10] F. Ardila and F. Block. Universal polynomials for Severi degrees of toric surfaces. Preprint, arXiv:1012.5305, 2010.

[Ber75] D. N. Bernstein. The number of roots of a system of equations. Funkcional. Anal. i Priložen., 9(3):1-4, 1975.

[BM07] E. Brugallé and G. Mikhalkin. Enumeration of curves via floor diagrams. C. R. Math. Acad. Sci. Paris, 345(6):329-334, 2007.

[BM09] E. Brugallé and G. Mikhalkin. Floor decompositions of tropical curves: the planar case. In Proceedings of Gökova Geometry-Topology Conference 2008, pages 64-90. Gökova Geometry/Topology Conference (GGT), Gökova, 2009.

[DFI95] P. Di Francesco and C. Itzykson. Quantum intersection rings. In The moduli space of curves (Texel Island, 1994), volume 129 of Progr. Math., pages 81-148. Birkhäuser Boston, Boston, MA, 1995.

[Enr12] F. Enriques. Sui moduli d'una classe di superficie e sul teorema d'esistenza per funzioni algebriche di due variabilis. Atti Accad. Sci. Torino, 47, 1912.

[FM10] S. Fomin and G. Mikhalkin. Labeled floor diagrams for plane curves. J. Eur. Math. Soc. (JEMS), 12(6):1453-1496, 2010.

[Ful84] W. Fulton. Introduction to intersection theory in algebraic geometry, volume 54 of CBMS Regional Conference Series in Mathematics. Published for the Conference Board of the Mathematical Sciences, Washington, DC, 1984.

[Göt98] L. Göttsche. A conjectural generating function for numbers of curves on surfaces. Comm. Math. Phys., 196(3):523-533, 1998.

[Har86] J. Harris. On the Severi problem. Invent. Math., 84(3):445-461, 1986.

[KP04] S. Kleiman and R. Piene. Node polynomials for families: methods and applications. Math. Nachr., 271:69-90, 2004.

[Pos09] A. Postnikov. Permutohedra, associahedra, and beyond. Int. Math. Res. Notices, pages 1026$1106,2009$.

[PRW08] A. Postnikov, V. Reiner, and L. Williams. Faces of generalized permutohedra. Documenta Math., 13:207-273, 2008.

[Sev21] F. Severi. Vorlesungen über Algebraische Geometrie. Teubner, Leipzig, 1921.

[Tze10] Y.-J. Tzeng. A proof of Göttsche-Yau-Zaslow formula. Preprint, arXiv:1009.5371, 2010. 